\title{
Invasive procedures in patients undergoing treatment with lenvatinib for thyroid cancer
}

\author{
SOJI TODA ${ }^{1}$, HIROYUKI IWASAKI ${ }^{1}$, DAISUKE MURAYAMA ${ }^{1}$, HIROTAKA NAKAYAMA ${ }^{2}$, \\ NOBUYASU SUGANUMA ${ }^{1,3}$ and KATSUHIKO MASUDO ${ }^{4}$
}

\author{
${ }^{1}$ Department of Breast and Endocrine Surgery, Kanagawa Cancer Center, Yokohama, Kanagawa 241-8515; \\ ${ }^{2}$ Department of Surgery, Hiratsuka Kyosai Hospital, Hiratsuka, Kanagawa 254-8502; ${ }^{3}$ Departments of Surgery, \\ Yokohama City University School of Medicine, Yokohama, Kanagawa 236-0004; ${ }^{4}$ Departments of Breast and \\ Thyroid Surgery, Yokohama City University Medical Center, Yokohama, Kanagawa 232-0024, Japan
}

Received August 21, 2020; Accepted February 9, 2021

DOI: $10.3892 / \mathrm{mco} .2021 .2243$

\begin{abstract}
Lenvatinib is a multi-tyrosine kinase inhibitor that inhibits angiogenesis and is currently in use for the treatment of refractory thyroid cancer. Therapy using this agent can be prolonged in patients, although serious complications may ensue among those who require surgical procedures. To the best of our knowledge, the safety of invasive surgical procedures in patients undergoing treatment with lenvatinib has not been fully evaluated. A total of 94 patients were treated with lenvatinib for thyroid cancer between June 2015 and August 2019 at the Kanagawa Cancer Center. Of this cohort, 14 invasive procedures were performed on 11 patients. A total of 8 of these procedures were performed under local anesthesia and 6 under general anesthesia; 3 belonging to the latter group were emergency procedures. No primary wound complications were observed among the patients in the cohort; however, one case of delayed healing secondary to placement of a thoracic drain for acute pneumothorax was reported in the present study. Lenvatinib was initially discontinued in this patient, but it was reintroduced 17 days later due to hypoxemia that may have been related to lung metastases. Hypoxemia improved, although wound healing spanned 14 weeks. Therapy was discontinued prior to percutaneous endoscopic gastrostomy in a patient with local progression; while no wound complications were observed, the patient ultimately died from exacerbation of neoplastic disease. In the current study, which focused on surgical interventions performed on patients undergoing lenvatinib treatment, 1 in 14 wound complications improved conservatively. However, it is critical
\end{abstract}

Correspondence to: Dr Soji Toda, Department of Breast and Endocrine Surgery, Kanagawa Cancer Center, 2-3-2 Nakao, Asahi-ku, Yokohama, Kanagawa 241-8515, Japan

E-mail: s-toda@kcch.jp

Key words: lenvatinib, vascular endothelial growth factor, percutaneous coronary angioplasty, surgical wound, invasive procedure to recognize that disease progression may occur if drugs are withdrawn prior to performing invasive procedures.

\section{Introduction}

Lenvatinib is a multi-kinase inhibitor that inhibits signaling via vascular endothelial growth factor (VEGF) receptors 1-3, fibroblast growth factor receptors 1-4, platelet-derived growth factor receptor- $\alpha$, and the ret and KIT proto-oncogene receptor tyrosine kinases; its overall antitumor effect relates to its capacity to inhibit angiogenesis $(1,2)$. In a phase III study of radioiodine-refractory differentiated thyroid carcinoma (DTC, SELECT study), lenvatinib treatment significantly improved the progression-free survival of patients compared with placebo (3). In a further subanalysis of the SELECT study, the overall survival was improved in age of over 65 years group (4). More recently, in a limited series of patients with stage IVC anaplastic thyroid cancer (ATC), the lenvatinib group had a better survival benefit than the palliative therapy group (5).

According to the real-world data of lenvatinib treatment for radioiodine-refractory DTC in Italy, 26 out of 82 patients experienced serious adverse events (SAEs); among the SAEs, five were drug related, including tracheal bleeding, pulmonary embolism, tracheoesophageal fistula, wound healing impairment, and cardiac flutter (6). In real-world data of France, in which 75 patients were analyzed, the incidence of treatment-related adverse events in all grades was $95 \%$, whereas that in grade 3 or higher was $48 \%$. SAEs were observed in 12 patients (16\%); 2 patients developed pneumothorax, 1 died, and the others required continued chest tube drainage (7).

Moreover, complications such as upper airway fistula formation and bleeding resulted in death in a small cohort of patients $(8,9)$. Of particular note, ongoing treatment with anti-VEGF inhibitors frequently results in wound complications due to inhibition of angiogenesis $(10,11)$. Lenvatinib therapy is often prolonged in patients with radioiodinerefractory DTC; a subset of these patients may ultimately experience medical conditions or complications require invasive procedures. There have been no studies that focus on the safety of invasive procedures in patients treated with 
lenvatinib. As such, we designed a study to address this issue among patients undergoing lenvatinib treatment at our hospital.

\section{Patients and methods}

Patients' selection and characteristics. Between June 2015 and August 2019, 82 patients received lenvatinib for thyroid cancer at Kanagawa Cancer Center. Among them, 58 had DTC, whereas 24 had ATC. All eligible participants provided informed consent. This study is approved by the institutional review board of Kanagawa Cancer Center (IRB approval number 27-61). Within this cohort, we identified 11 patients who required invasive procedures while undergoing treatment with lenvatinib. Patients in this cohort included those involving general or local anesthesia; those reporting minor procedures including simple sutures or dental procedures were excluded from the study. Eleven patients (6 males and 5 females) underwent 14 invasive procedures; the median age of the patients in this cohort was 72 (range, 59 to 83) years. The histological thyroid cancer diagnoses included papillary carcinoma $(n=8)$, follicular carcinoma $(n=2)$, and ATC $(n=1)$. In addition, three patients had diabetes mellitus, and two were taking anticoagulants.

Evaluation of the nutrition status. For evaluating the nutrition status, we calculated the prognostic nutritional index (PNI): PNI=10 Alb. +0.005 Lymph. C., where Alb. is the serum albumin level $(\mathrm{g} / 100 \mathrm{ml})$ and Lymph. C. is the total lymphocytes count $/ \mathrm{mm}^{3}$ of peripheral blood. When the index is below 40, resection and anastomosis of gastrointestinal tract may be contraindicated because of the risk of wound complication (12). Unfortunately, we have not acquired the data of total cholesterol level required for CONUT. Hence, we evaluated the nutritional status instead using the prognostic nutritional index. Four patients had a PNI score of below 40 (Table I). We examined the postsurgical course, including lenvatinib dose, preprocedure withdrawal period, and wound complication reports for each patient.

\section{Results}

Invasive procedure characteristics. Of the 14 invasive procedures performed on patients with thyroid cancer undergoing treatment with lenvatinib, four were for thoracic drain placement. Eight of the procedures that were performed under local anesthesia included construction of a port for a central venous catheter, cataract surgery, percutaneous endoscopic gastrostomy, percutaneous coronary angioplasty (PCI), and the aforementioned thoracic drainage. Six of the procedures performed under general anesthesia included cerebral aneurysm clipping, total thyroidectomy with resection of the upper mediastinal tumor, laparotomy for gastrostomy, radical inguinal hernia repair, appendectomy, and cholecystectomy. Of all the procedures, the PCI, appendectomy, and cholecystectomy were performed on an emergency basis. Meanwhile, total thyroidectomy with resection of the upper mediastinal tumor was performed in a 75-year-old woman. In this case (13), which we had reported previously, the tumor had invaded the internal jugular and subclavian veins, forming a tumor embolus in the right brachiocephalic vein and reaching the vicinity of the superior vena cava. In addition, for life-saving purposes, we used lenvatinib. Consequently, the tumor shrunk after 4 months, and surgery was performed thereafter.

Lenvatinib administration status for each invasive procedure. The mean period between initiation of lenvatinib therapy and the performance of an invasive procedure was 393 days (range, 29 to 1,318 days). Doses taken during the period prior to surgery included $10,12,14$, and $20 \mathrm{mg}$. Three of the patients (21.4\%) underwent a withdrawal period of 1-2 weeks prior to surgery; eight of MCO-15315-261201_Toda MCO-15315261201_Toda the patients (57.1\%) remained on lenvatinib while undergoing the invasive procedure. Lenvatinib therapy had been withdrawn from the three cases in the first group (21.4\%) for unrelated reasons, i.e., due to previous adverse events or to facilitate other forms of treatment. In all cases, lenvatinib treatment was resumed after confirming appropriate postsurgical wound healing; four of the patients (28.6\%) underwent no withdrawal whatsoever. Among those who stopped therapy prior to surgery, $(42.8 \%)$ resumed within 3 weeks, while $7.1 \%$ (one case) resumed treatment after more than 3 weeks; lenvatinib therapy was not reintroduced in three of the patient cases (28.6\%; Table II).

Adverse events during invasive procedures. Most of the patients experienced no postoperative wound complications. We observed delayed wound healing in one patient secondary to a 20 Fr. trocar catheter thoracic drain placed for the treatment of a left pneumothorax. In this case, lenvatinib had been discontinued on the day of the procedure. On day 5 postprocedure, pleurodesis was performed; the catheter was removed on postoperative day 11. The patient subsequently developed hypoxemia which may have been related to enlarging lung metastases; lenvatinib treatment was resumed on day 17. This intervention resulted in improved oxygenation, although the wound took 14 weeks to heal (Fig. 1).

No other wound complications were identified in this patient cohort, although one patient died of disease progression during the period of lenvatinib withdrawal. He had been undergoing treatment with lenvatinib for multiple lung metastases and local recurrences, had undergoing repeated bouts of drug withdrawal and resumption due to concerns regarding an open leg ulcer. This patient required a gastrostomy to treat esophageal stenosis due to local recurrence that caused difficulty with swallowing. He was withdrawn from lenvatinib after the endoscopic gastrostomy was performed, but died on postoperative day 8 due to pleural effusion and general deterioration of his general. The surgical wound was healing appropriately with no abnormalities.

\section{Discussion}

VEGF-targeted inhibitors of angiogenesis have been associated with an increased risk of wound complications (14). In rats, treatment with the tyrosine kinase inhibitor, semaxanib, resulted in reduced tissue perfusion and micro-vessel density, but it did not impair wound healing (15). Other studies have revealed that administration of bevacizumab resulted in reduced wound strength and wound healing rates in a macaque model (16). 
Table I. Patient demographics.

\begin{tabular}{lc}
\hline Characteristic & $\mathrm{n}=11$ \\
\hline Median age (range), years & $72(59-83)$ \\
Sex, $\mathrm{n}(\%)$ & \\
Male & $6(54.5)$ \\
Female & $5(45.5)$ \\
Pathological type of thyroid cancer, $\mathrm{n}(\%)$ & \\
Papillary carcinoma & $8(72.7)$ \\
Follicular carcinoma & $2(18.2)$ \\
Anaplastic carcinoma & $1(9.1)$ \\
Eastern Cooperative Oncology Group & \\
Performance status, $\mathrm{n}(\%)$ & \\
0 & $9(81.8)$ \\
1 & $2(18.2)$ \\
Site of metastasis, $\mathrm{n}(\%)$ & \\
Lung & $7(63.6)$ \\
Bone & $4(36.4)$ \\
Lymph node & $4(36.4)$ \\
Wound healing factors, $\mathrm{n}(\%)$ & \\
Diabetes mellitus & $3(27.3)$ \\
Anti-coagulation medicine & $2(18.2)$ \\
Prognostic nutritional index, $\mathrm{n}(\%)$ & \\
$>40$ & \\
$\leq 40$ & \\
\hline
\end{tabular}

VEGF inhibitors include bevacizumab and ramucirumab, which are humanized monoclonal antibodies that target VEGF and VEGFR, respectively. Similarly, aflibercept has direct inhibitory effects on VEGF-A, VEGF-B and placental growth factor (PIGF) and low molecular weight multi-tyrosine kinase inhibitors (mTKIs), including sorafenib and lenvatinib, inhibit intracellular tyrosine kinase activity VEGFR-activated cells. Bevacizumab has a half-life of 20 days, while the half-life of lenvatinib is $35.4 \mathrm{~h}$; the half-lives of other TKIs range from 1-3 days, while mTKIs have a relatively short half-life (Table III) (17-22).

Administration of bevacizumab results in an increased the risk of postsurgical wound complications. In a phase III trial (NSABP C-08), adjuvant therapy with bevacizumab that was initiated at 29-50 days after surgery for colon cancer surgery, was associated with an increased frequency of wound complications at grade 3 or higher (10). A retrospective analysis of cases in which emergency surgery was required among patients in trials and undergoing treatment with bevacizumab for stage III colorectal cancer revealed that wound complications (grade 3 or higher) occurred more frequently in the bevacizumab group (10/75, or $13 \%)$ compared to only $3.4 \%(1 / 29)$ in the among those receiving standard chemotherapy alone (11).

By contrast, completely different results were obtained from studies in which preoperative treatment with mTKIs were evaluated prior to surgical intervention for renal cell carcinoma. For example, Cowey et al performed laparoscopic or open nephrectomy on 30 patients that were undergoing treatment with sorafenib (23). The surgical complications
Table II. Lenvatinib administration status for each invasive procedure.

\begin{tabular}{lc}
\hline Lenvatinib administration status & $\mathrm{n}(\mathrm{n}=14)$ \\
\hline $\begin{array}{l}\text { Period from start of lenvatinib to } \\
\text { surgical procedure (days) }\end{array}$ & 393 \\
Dosage of lenvatinib prior to & (range 29-1,318) \\
procedure (mg/per day) & \\
10 & $3(21.4)$ \\
12 & $2(14.3)$ \\
14 & $4(28.6)$ \\
20 & $5(35.7)$ \\
Withdrawal period prior to & \\
procedure (weeks) & \\
None & $8(57.1)$ \\
1 & $1(7.1)$ \\
2 & $2(14.3)$ \\
$\geq 3$ & $3(21.4)$ \\
Period from time of procedure & \\
to reintroduction of lenvatinib & \\
No withdrawal & \\
Within 7 days & \\
$8-21$ days & $4(28.6)$ \\
$\geq 22$ days & $3(21.4)$ \\
Did not resume & $3(21.4)$ \\
\hline
\end{tabular}

Data are presented as n (\%) unless otherwise specified.

included only one case of a superficial wound break. The preoperative withdrawal period among those patients who underwent sorafenib treatment was 3 days (range 2-14 days); among the five patients identified with distant metastases, TKI treatment was reintroduced 4-6 weeks after surgery when wound healing was confirmed. Similarly, Zhang et al evaluated the impact of preoperative treatment with sorafenib in 18 patients; only one patient experienced postoperative bleeding (24). The median preoperative drug withdrawal period was 12 days (range, 7-30 days) and sorafenib treatments were resumed within 2-4 weeks after surgery for seven patients with distant metastases.

In the aforementioned studies, patients had undergone sorafenib treatment prior to major surgery; the frequency of wound complications was low in most cases. Lenvatinib is also to be considered safe for this application, as it has a similar half-life and mechanism of action. However, due to the small number of cases that have been reviewed, the decision to perform invasive treatments should be undertaken carefully.

Wound healing with low-dose lenvatinib treatment has also been previously reported. Resteghini et al presented a case of a locally advanced DTC. In this case, lenvatinib was administered at a standard dose of $24 \mathrm{mg}$. Although it had a dramatic effect, it formed an ulcer because of tumor necrosis, causing s-evere tracheal bleeding. Therefore, lenvatinib was resumed after a 3 -week interval from the last dose. Treatment dosing and local bleeding control were achieved by progressively tapering down lenvatinib to $14 \mathrm{mg}(25)$. 
Table III. Half-lives of VEGF inhibitors in vivo.

\begin{tabular}{llc}
\hline Drug & \multicolumn{1}{c}{ Activity } & Half-life (reference) \\
\hline Bevacizumab & Antibody against VEGF-A & 20 days $(11)$ \\
Ramucirumab & Antibody against VEGFR-2 & 8 days $(12)$ \\
Aflibercept & Inhibitor of VEGF-A, VEGF-B and PlGF & $5.59 \mathrm{days}(13)$ \\
Sorafenib & Inhibits the tyrosine kinase activity of VEGFRs & $28.1 \mathrm{~h}(14)$ \\
Lenvatinib & Inhibits the tyrosine kinase activity of VEGFRs & $35.4 \mathrm{~h}(15)$ \\
Sunitib & Inhibits the tyrosine kinase activity of VEGFRs & $41-86 \mathrm{~h}(16)$
\end{tabular}

P1GF, placental growth factor.

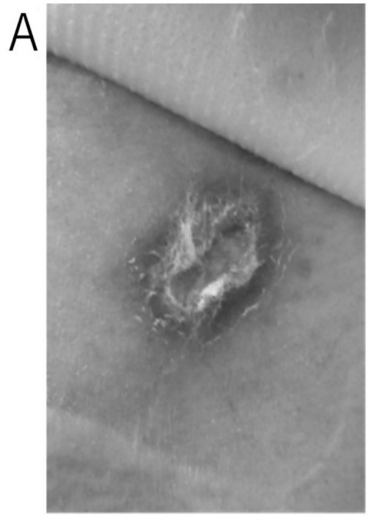

6 weeks

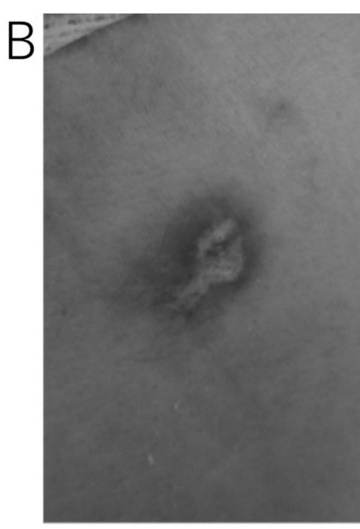

10 weeks

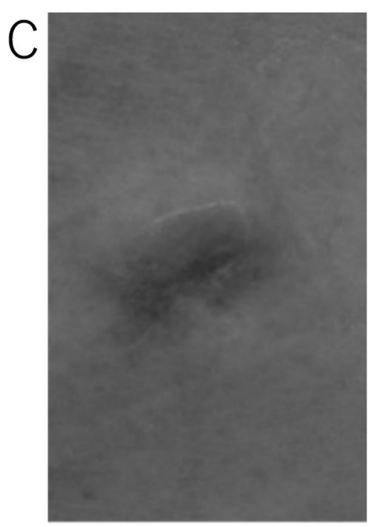

14 weeks

Figure 1. Delayed wound healing in a patient with a thoracic drain placed for the treatment of pneumothorax. Images depict the extent of healing at (A) 6 , (B) 10 and (C) 14 weeks after removal of the catheter.

We identified one patient in which the overall condition deteriorated rapidly after lenvatinib was discontinued. It is clear that TKI withdrawal facilitates tumor growth. VEGF inhibition with a TKI for a period of 7 days results in 50-60\% loss of the tumor vasculature; however, tumor vessels can regenerate within 7 days after drug administration has been discontinued (26). As such, it is critical to consider this point among patients with DTC when considering acute withdrawal of lenvatinib from patients who are maintained on long-term regimens.

Most invasive procedures in patients undergoing treatment with lenvatinib did not result in postsurgical wound complications. Current recommendations suggest that patients should be withdrawn from this drug for a period of 1-2 weeks prior to scheduled surgical procedures and that therapy can be resumed after postoperative wound healing has been confirmed. However, this approach requires caution as it is critical to consider the possibility of significant disease progression during the course of an extended drug holiday.

\section{Acknowledgements}

Not applicable.

\section{Funding}

No funding was received.

\section{Availability of data and materials}

The datasets used and/or analyzed during the current study are available from the corresponding author on reasonable request.

\section{Authors' contributions}

ST, HI, and NS designed the current study. HN analyzed the data. HI, NS, DM and KM performed the surgery and provided patient care. DM and KM contributed to patient data acquisition. ST and HI confirm the authenticity of all the raw data. All authors read and approved the final manuscript.

\section{Ethics approval and consent to participate}

All procedures involving human participants were conducted in accordance with the ethical standards of the institutional and/or national research committee and the tenets of the 1964 Helsinki Declaration and its later amendments or comparable ethical standards. The current study followed the 'Ethical Guidelines for Medical Research on Human Subjects' and conducted the present study with approval from the institutional review board of Kanagawa Cancer Center (approval no. 27-61). Informed consent was obtained from all individual participants included in the study. 


\section{Patient consent for publication}

All patients provided a comprehensive consent form stating that personal data could be used for academic presentation or paper presentation while ensuring complete anonymity before receiving the treatment.

\section{Competing interests}

The authors declare that they have no competing interests.

\section{References}

1. Matsui J, Funahashi Y, Uenaka T, Watanabe T, Tsuruoka A and Asada M: Multi-kinase inhibitor E7080 suppresses lymph node and lung metastases of human mammary breast tumor MDA-MB-231 via inhibition of vascular endothelial growth factor-receptor (VEGF-R) 2 and VEGF- R3 kinase. Clin Cancer Res 14: 5459-5465, 2008.

2. Matsui J, Yamamoto Y, Funahashi Y, Tsuruoka A, Watanabe T, Wakabayashi T, Uenaka T and Asada M: E7080, a novel inhibitor that targets multiple kinases, has potent antitumor activities against stem cell factor producing human small cell lung cancer H146, based on angiogenesis inhibition. Int J Cancer 122: 664-671, 2008

3. Schlumberger M, Tahara M, Wirth LJ, Robinson B, Brose MS, Elisei R, Habra MA, Newbold K, Shah MH, Hoff AO, et al Lenvatinib versus placebo in radioiodine-refractory thyroid cancer. N Engl J Med 372: 621-630, 2015.

4. Brose MS, Worden FP, Newbold KL, Guo M and Hurria A: Effect of age on the efficacy and safety of lenvatinib in radioiodine-refractory differentiated thyroid cancer in the Phase III SELECT Trial. J Clin Oncol 35: 2692-2699, 2017.

5. Iwasaki H, Toda S, Suganuma N, Murayama D, Nakayama $H$ and Masudo K: Lenvatinib vs. palliative therapy for stage IVC anaplastic thyroid cancer. Mol Clin Oncol 12: 138-143, 2020.

6. Locati LD, Piovesan A, Durante C, Bregni M, Castagna MG Zovato S, Giusti M, Ibrahim T, Puxeddu E, Fedele G, et al: Real-world efficacy and safety of lenvatinib: data from a compassionate use in the treatment of radioactive iodine-refractory differentiated thyroid cancer patients in Italy. Eur J Cancer 118: 35-40, 2019.

7. Berdelou A, Borget I, Godbert Y, Nguyen T, Garcia ME, Chougnet CN, Ferru A, Buffet C, Chabre O, Huillard O, et al: Lenvatinib for the treatment of radioiodine-refractory thyroid cancer in real-life practice. Thyroid 28: 72-78, 2018.

8. Blevins DP, Dadu R, Hu M, Baik C, Balachandran D, Ross W, Gunn B and Cabanillas ME: Aerodigestive fistula formation as a rare side effect of antiangiogenic tyrosine kinase inhibitor therapy for thyroid cancer. Thyroid 24: 918-922, 2014.

9. Iwasaki H, Yamazaki H, Takasaki H, Suganuma, N Nakayama H, Toda S and Masudo K: Lenvatinib as a novel treatment for anaplastic thyroid cancer: A retrospective study. Oncol Lett 16: 7271-7277, 2018.

10. Allegra CJ, Yothers G, O'Connell MJ, Sharif S, Colangelo LH, Lopa SH, Petrelli NJ, Goldberg RM, Atkins JN, Seay TE, et al: Initial safety report of NSABP C-08: A randomized phase III study of modified FOLFOX6 with or without bevacizumab for the adjuvant treatment of patients with stage II or III colon cancer. J Clin Oncol 27: 3385-3390, 2009.

11. Scappaticci FA, Fehrenbacher L, Cartwright T, Hainsworth JD, Heim W, Berlin J, Kabbinavar F, Novotny W, Sarkar S and Hurwitz H: Surgical wound healing complications in metastatic colorectal cancer patients treated with bevacizumab. J Surg Oncol 91: 173-180, 2005.
12. Onodera T, Goseki N and Kosaki G: Prognostic nutritional index in gastrointestinal surgery of malnourished cancer patients. Nihon Geka Gakkai Zasshi 85: 1001-1005, 1984 (In Japanese).

13. Iwasaki H, Toda S, Ito H, Nemoto D, Murayama D, Okubo Y, Hayashi $\mathrm{H}$ and Yokose T: A case of unresectable papillary thyroid carcinoma treated with lenvatinib as neoadjuvant chemotherapy. Case Rep Endocrinol 2020: 6438352, 2020.

14. Chen HX and Cleck JN: Adverse effects of anticancer agents that target the VEGF pathway. Nat Rev Clin Oncol 6: 465-477, 2009.

15. Roman CD, Choy H, Nanney L, Riordan C, Parman K, Johnson D and Beauchamp RD: Vascular endothelial growth factor-mediated angiogenesis inhibition and postoperative wound healing in rats. J Surg Res 105: 43-47, 2002.

16. Cornacoff JB, Howk K, Pikounis B, Mendenhall V and Martin P: Development of a method for the evaluation of wound tensile strength in cynomolgus macaques. J Pharmacol Toxicol Methods 57: 74-79, 2008.

17. Lu JF, Bruno R, Eppler S, Novotny W, Lum B and Gaudreault J: Clinical pharmacokinetics of bevacizumab in patients with solid tumors. Cancer Chemother Pharmacol 62: 779-786, 2008.

18. Yamaguchi K, Fujitani K, Nagashima F, Omuro Y, Machida N, Nishina T, Koue T, Tsujimoto M, Maeda K and Satoh T: Ramucirumab for the treatment of metastatic gastric or gastroesophageal junction adenocarcinoma following disease progression on first-line platinum- or fluoropyrimidine-containing combination therapy in Japanese patients: A phase 2, open-label study. Gastric Cancer 21: 1041-1049, 2018

19. Yoshino T, Yamazaki K, Yamaguchi K, Doi T, Boku N, Machida N, Onozawa Y, Asayama M, Fujino T and Ohtsu A: A phase I study of intravenous aflibercept with FOLFIRI in Japanese patients with previously treated metastatic colorectal cancer. Invest New Drug; 31: 910-917, 2013.

20. Strumberg D, Richly H, Hilger RA, Schleucher N, Korfee S, Tewes M, Faghih M, Brendel E, Voliotis D, Haase CG, et al: Phase I clinical and pharmacokinetic study of the Novel Raf kinase and vascular endothelial growth factor receptor inhibitor BAY 43-9006 in patients with advanced refractory solid tumors. J Clin Oncol 23: 965-972, 2005.

21. Dubbelman AC, Rosing H, Nijenhuis C, Huitema ADR, Mergui-Roelvink M, Gupta A, Verbel D, Thompson G, Shumaker R, Schellens JH and Beijnen JH: Pharmacokinetics and excretion of (14) C-lenvatinib in patients with advanced solid tumors or lymphomas. Invest New Drug 33: 233-240, 2015.

22. Faivre S, Delbaldo C, Vera K, Robert C, Lozahic S, Lassau N, Bello C, Deprimo S, Brega N, Massimini G, et al: Safety, pharmacokinetic, and antitumor activity of SU11248, a novel oral multitarget tyrosine kinase inhibitor, in patients with cancer. J Clin Oncol 24: 25-35, 2006

23. Cowey CL, Amin C, Pruthi RS, Wallen EM, Nielsen ME, Grigson G, Watkins C, Nance KV, Crane J, Jalkut M, et al: Neoadjuvant clinical trial with sorafenib for patients with stage II or higher renal cell carcinoma. J Clin Oncol 28: 1502-1507, 2010.

24. Zhang Y, Li Y, Deng J, Ji Z, Yu H and Li H: Sorafenib neoadjuvant therapy in the treatment of high risk renal cell carcinoma. PLoS One 10: E0115896, 2015.

25. Resteghini C, Locati LD, Bossi P, Bergamini C, Guzzo M and Licitra L: Do not throw the baby out with the bathwater: SELECT a personalized, de-escalated lenvatinib schedule allows response in locally advanced DTC while controlling major drug-related bleeding. Ann Oncol 28: 2321-2322, 2017.

26. Mancuso MR, Davis R, Norberg SM, O'Brien S, Sennino B, Nakahara T, Yao VJ, Inai T, Brooks P, Freimark B, et al: Rapid vascular regrowth in tumors after reversal of VEGF inhibition. J Clin Invest 116: 2610-2621, 2006.

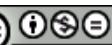

This work is licensed under a Creative Commons Attribution-NonCommercial-NoDerivatives 4.0 International (CC BY-NC-ND 4.0) License. 